\title{
LA VEGETACIÓN DE LAS GEOFORMAS PROGLACIARES EN LOS GLACIARES BALMACEDA, TYNDALL, TARABA Y EMA, MAGALLANES, CHILE
}

\author{
PROGLACIAL LANDFORMS VEGETATION IN THE BALMACEDA, \\ TYNDALL, TARABA AND EMA GLACIERS, MAGALLANES, CHILE
}

Orlando Dollenz", Juan Henriquez" \& Erwin Domínguez"***

\begin{abstract}
The forms and the type of substrate that constitutes the proglacial landforms determine the type of vegetation that colonizes them. In this article, the vegetation that covers proglacial landforms in four glacier valleys of the region of Magallanes are described, analyzing the floristic composition and species richness on the landforms of each glacier. One determined that the succession goes towards the climax of the forests of Nothofagus in the substrates with good drainage, moraines, alluviums, lacustrine terraces, or towards the climax of the ciperaceous moorland or Sphagnum bog in the substrates with deficient drainage, like depressions between moraines with clays, or moraine material with clays locked up between moraines arcs. In valleys glaciers the greater species richness tends to be in bottom moraines, due to the existence of numerous microsites. It is ratified that the first tree species that colonize on glacial valleys are Nothofagus betuloides, N. antarctica and Embothrium coccineum.
\end{abstract}

Key word: Plant colonization, Southern Ice Field, Patagonia.

\section{RESUMEN}

Las geoformas proglaciares sus formas y el tipo de sustrato que las constituyen determinan el tipo de vegetación que las coloniza. En este artículo se describe la vegetación que cubre las geoformas proglaciares en cuatro valles glaciares de la región de Magallanes, analizando la composición florística y la

* Facultad de Ciencias, Universidad de Magallanes.

* Instituto de la Patagonia, Universidad de Magallanes.

$*$ Instituto Nacional de Investigaciones Agropecuarias, INIA-Magallanes. 
distribución de la riqueza de especies en las geoformas de cada glaciar. Se determinó que la sucesión se dirige hacia el climax de los bosques de Nothofagus en los sustratos con buen drenaje, morrenas, aluviones, terrazas lacustres, o hacia el climax de las turberas de ciperáceas o de Sphagnum en los sustratos con drenaje deficiente como depresiones intermorrénicas con arcillas, o material morrénico con arcillas encerrado entre arcos morrénicos. En los valles glaciares la riqueza de especies mayor tiende a estar en las morrenas de fondo, debido a la existencia de numerosos micrositios. Se ratifica que las primeras especies arbóreas que se establecen en los valles glaciales son Nothofagus betuloides, N. antarctica y Embothrium coccineum.

Palabras clave: Colonización vegetal, Campo de Hielo sur, Patagonia.

\section{INTRODUCCIÓN}

Los estudios publicados de la vegetación en los valles glaciares de Magallanes tienen objetivos como el establecimiento arbóreo (Pisano 1978), la estructura de edades y dinámica de los bosques periglaciares (Armesto et al., 1992), la colonización vegetal y fitogeografía (Moore \& Pisano, 1997), el análisis florístico de los valles glaciares (Henríquez, 2002), estudios liquenométricos (Henríquez \& Caamaño, 2002), y la descripción de la vegetación que cubre las geoformas proglaciares (Pisano, 1971; Dollenz, 1991; Domínguez et al., 1999).

Las geoformas proglaciares fundamentales y en sus distintos tipos son las morrenas, los depósitos fluvioglaciales y las rocas planas o en pendiente (Aniya, 1985; Burrows, 1990; Domínguez et al., 1999). Estas geoformas se caracterizan por sus formas y el tipo de sustrato, las rocas consolidadas y las no consolidadas, como los aluviones de arenas y gravas, las morrenas compuestas de piedras, arenas, limos, arcillas y el tipo de drenaje, estos factores en conjunto determinan el tipo de vegetación que las coloniza. Otros factores ambientales que influyen también en el proceso de colonización vegetal son el tiempo, la exposición del sitio, el clima local y la distancia de las fuentes de semillas.

En este articulo se describe la vegetación que cubre las geoformas proglaciares de cuatro glaciares de la región de Magallanes, el glaciar Tyndall, el Balmaceda y el Taraba ubicados en el Campo de Hielo Sur, y el glaciar Ema ubicado en la cordillera Darwin, Andes de Tierra del Fuego (Fig. 1) cuyas geoformas se describen en Strelin et al. (2008). Se analiza también la distribución de la riqueza de especies en las geoformas de cada glaciar.

\section{MATERIALES Y MÉTODOS}

Los cuatro glaciares estudiados en la región de Magallanes son: el Tyndall (51 $\left.11^{\circ} \mathrm{S}, 73^{\circ} 11^{\prime} \mathrm{O}\right)$, el

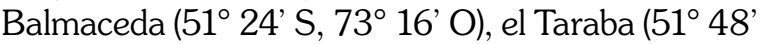
$\mathrm{S}, 73^{\circ} 29^{\prime}$ O) en el Campo de Hielo Sur y el Ema ( $54^{\circ} 27^{\prime} \mathrm{S}, 70^{\circ} 42^{\prime} \mathrm{O}$ ) en la cordillera Darwin de Tierra del Fuego (Fig. 1). Las fechas en que fueron visitados son: el Tyndall en noviembre de 1994, el Ema en diciembre de 1994, el Balmaceda en enero de 1996 y el Taraba en enero de 1997.

No existen estaciones climáticas en los sitios estudiados, pero (Fujiyoshi, et al. 1987) registró una precipitación anual de $3.700 \mathrm{~mm}$ para el glaciar San Rafael en el Campo de Hielo Norte y estima que la precipitación en el Campo de Hielo puede ser tres veces mayor que la del pie del glaciar, unos 10.000 $\mathrm{mm}$ anuales. Por otra parte, las isoyetas de Jerez y Arancibia, (1972) estiman una precipitación entre 2.000 a $3.000 \mathrm{~mm}$ anuales para los glaciares en estudio, lo que indicaría, siguiendo a (Fujiyoshi et. al 1987), una precipitación estimada en el Campo de Hielo Sur entre 6.000 y $9.000 \mathrm{~mm}$ anuales.

En cada una de las geoformas identificadas se realizaron varios relevamientos de la vegetación de acuerdo al método de Braun Blanquet (MuellerDombois y Ellenberg 1974), en que cada parcela de muestreo debe ser homogénea en su fisonomía o aspecto vegetacional y en sus condiciones ambientales. Estas parcelas de 5 × $5 \mathrm{~m}(25 \mathrm{~m} 2)$ sobrepasan el área mínima de muestreo para arbustos y formaciones herbáceas, y es ajustada para los bosques considerando la restringida disponibilidad de superficies homogéneas amplias en las geoformas.

Se tabuló para cada glaciar la composición florística de las geoformas (Tablas 1, 2, 3 y 4) promediando los valores de cobertura de las especies del 
conjunto de parcelas de cada geoforma identificada. A las matrices numéricas derivadas de las tablas se les aplicó un análisis de agrupamiento o de clasificación, utilizando el software ACOM (Navarro 1984) $e$ índice de Dice, para conocer la similitud florística entre las geoformas de cada glaciar.

Las colecciones botánicas se encuentran depositadas en el herbario del Instituto de la Patagonia (HIP), Universidad de Magallanes, y las plantas fueron determinadas por el botánico Ing. Agr. Edmundo Pisano.

\section{RESULTADOS}

Se describen las geoformas y la vegetación de los valles glaciares separadamente considerando que la sucesión vegetal en ellos, muy probablemente, son espacial y temporalmente distintas, dadas las múltiples variaciones de los factores locales que pueden influir. Además, el área del glaciar Tyndall tiene actual uso ganadero y señales de incendios de bosques antiguos; el área del glaciar Balmaceda tuvo un intento fracasado de colonización ganadera

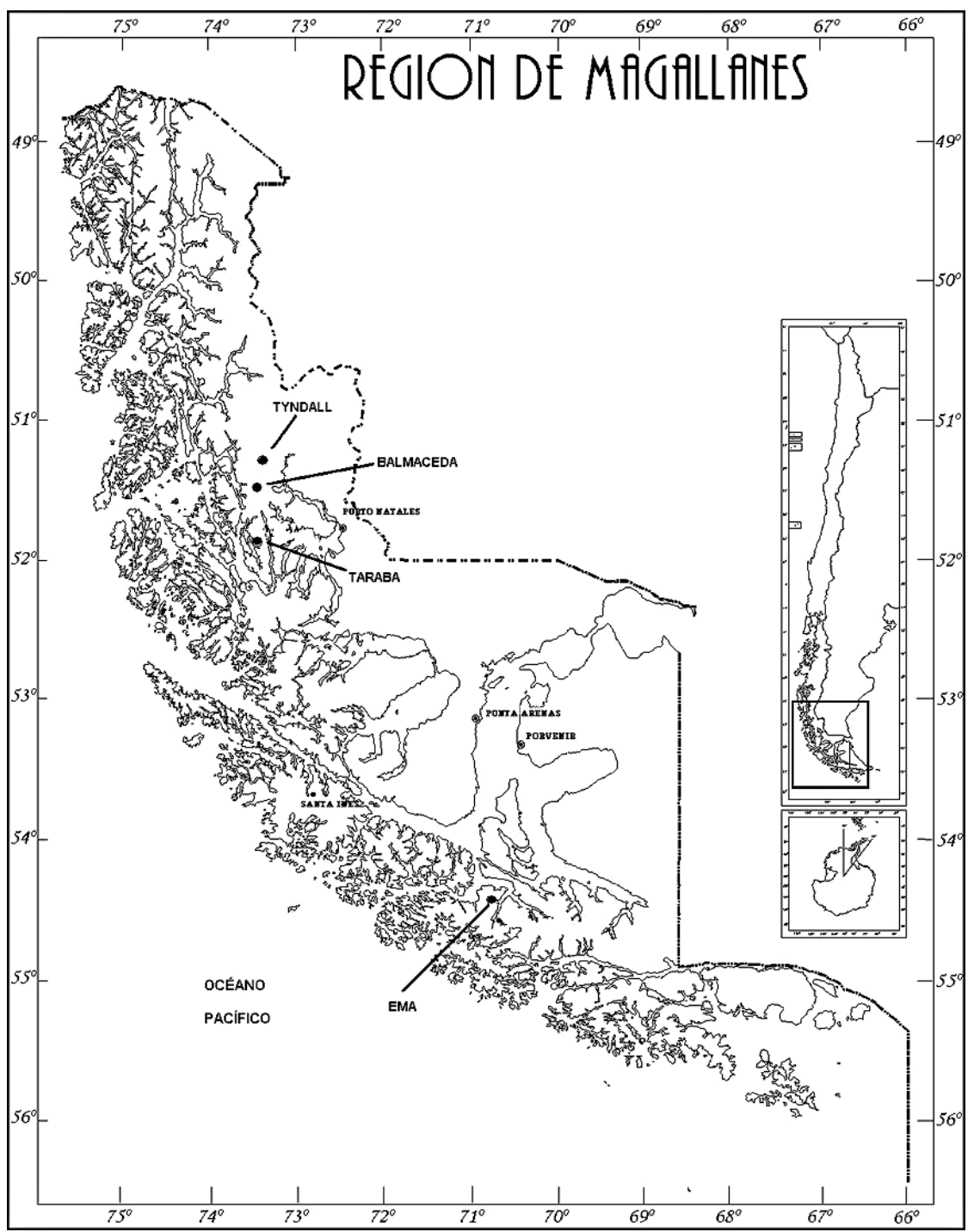

Fig. 1. Localización de los glaciares Tyndall ( $\left.51^{\circ} 15^{\prime} \mathrm{S}, 73^{\circ} 11^{\prime} \mathrm{O}\right)$, Balmaceda ( $\left.51^{\circ} 24^{\prime} \mathrm{S}, 73^{\circ} 16^{\prime} \mathrm{O}\right)$, Taraba $\left(51^{\circ} 48^{\prime} \mathrm{S}, 73^{\circ} 29^{\prime}\right.$ O) en el Campo de Hielo Sur y Ema (54 $27^{\prime}$ S, $70^{\circ} 42^{\prime}$ O) en la cordillera Darwin de Tierra del Fuego. 
en la primera mitad del siglo veinte sin impacto en el paisaje, no se encontraron especies vegetales introducidas; los glaciares Taraba y Ema pueden considerarse prístinos, su posición y características geográficas y su vegetación no favorecen por el momento las actividades humanas permanentes.

\section{GEOFORMAS PROGLACIARES DEL GLACIAR BALMACEDA Y SU VEGETACIÓN}

Morrenas laterales (Tabla 1, Bm1, Bm2)

En la morrena cuya pendiente cae al borde del lago glaciar (Tabla 1, Bm1), predomina un bosquecillo abierto de coihue de Magallanes, Nothofagus betuloides y ñire, $N$. antarctica con arbolitos de hasta 4 $m$ de altura y arbustos de chaura, Gaultheria mucronata. El piso está densamente cubierto por briófitas, helechos y una vegetación herbácea dominada por Gunnera magellanica y Anthoxanthum redolens.

En la pendiente opuesta al lago de la misma morrena y en los restantes arcos morrénicos sucesivos se encuentran bosques densos de coihue por ambas pendientes, con árboles de hasta $15 \mathrm{~m}$ de altura y hasta $40 \mathrm{~cm}$ de DAP. El estrato arbustivo es escaso con michay, Berberis ilicifolia y chaura, Gaultheria mucronata y trepadoras como Lebetanthus myrsinites (Tabla 1, Bm2). El piso está cubierto de briófitas y helechos y pocas especies herbáceas.

\section{Aluviones intermorrénicos (Tabla 1, Ba)}

En estos planos aluviales pedregosos, de gravas y arenas gruesas (Tabla $1, \mathrm{Ba}$ ) con buen drenaje, dominan bosquecillos de ñire, Nothofagus antarctica, con arbolitos de hasta $4 \mathrm{~m}$ de altura, y un estrato arbustivo muy denso de murtilla, Empetrum rubrum y briófitas.

Morrena de fondo (Tabla 1, Bf)

En el área adyacente al hielo, en gran parte desvegetada, se encuentran diversas especies herbáceas pioneras en pequeños manchones sobre material morrénico disperso y en grietas y depresiones rocosas húmedas (Tabla $1, \mathrm{Bf}$ ). Numerosas especies están solo presentes en este ambiente, Baccharis nivale, Deschampsia parvula, Nassauvia magellanica, Epilobium nivale, Calceolaria tenella,
Senecio cuneatus, Poa rigidifolia, Ourisia ruelloides. En sitios más alejados del hielo en depresiones, grietas y lagunillas aparecen pequeñas colonizaciones de turberas con junquillo, Marsippospermum grandiflorum y Carex banksii y pequeños árboles como coihues, Nothofagus betuloides y ñires, $N$. antarctica.

Planos rocosos (Tabla 1, Br1, Br2)

En las planicies rocosas externas a los arcos morrénicos, pulidas por los hielos, con suelos delgados, dominan las turberas de ciperáceas integradas por Schoenus antarcticus, Carpha schoenoides con la podocarpácea enana Lepidothamnus fonkii y de plantas pulvinadas como Astelia pumila, Donatia fascicularis (Tabla 1, Br1). Hay restos de bosquecillos de ciprés de las Guaitecas, Pilgerodendron uviferum explotados.

Se encuentran también turberas de esfagno, Sphagnum magellanicum, de reducida superficie (Tabla 1, Br2) con abundancia de murtilla, Empetrum rubrum y junquillo, Marsippospermum grandiflorum, y con colonización incipiente de coihue, Nothofagus betuloides.

Cerros rocosos (Tabla 1, Bc1, Bc2)

En las hondonadas y quebradas húmedas de los cerros de hasta $400 \mathrm{msnm}$, adyacentes al valle glaciar, domina el bosque de coihue, Nothofagus betuloides, con ciprés de las Guaitecas, Pilgerodendron uviferum, canelo, Drimys winteri, y fuinque, Lomatia ferrugínea (Tabla 1, Bc1). En el estrato arbustivo se encuentran el taique, Desfontainia fulgens y michay, Berberis ilicifolia, la trepadora coicopihue, Philesia magellanica y helechos como Blechnum magellanicum y Sticherus quadripartitus.

En las laderas de los cerros (Tabla 1, Bc2) también se encuentran turberas de ciperáceas con Schoenus antarcticus, Carpha schoenoides y junquillo, Marsippospermum grandiflorum. En algunas lagunas se encuentra la herbácea acuática arraigada con hojas flotantes Potamogeton linguatus.

Análisis de agrupamiento de la vegetación

En el dendrograma (Fig. 2) se separan las turberas de ciperáceas de Schoenus y Carpha 
que cubren en parte los planos y las laderas de los cerros rocosos externos al valle glacial (Tabla $1, \mathrm{Bc} 2, \mathrm{Br} 1$ ) con una similitud de un $73 \%$. Las otras comunidades se agrupan escalonadamente entre un 56 a un $24 \%$ de similitud florística. Estas fisonómicamente corresponden a los bosques de coihue de las morrenas (Tabla 1, Bm1, Bm2) y de los cerros rocosos (Tabla 1, Bc1), los bosquecillos de ñire de los aluviones (Tabla 1, Ba), la turba de esfagno de planos rocosos con drenaje pobre (Tabla 1, Br2), y a la morrena de fondo (Tabla 1, $\mathrm{Bf}$ ) en gran parte desvegetada con colonizaciones herbáceas, turbosas y arbóreas.

La riqueza de especies

Las geoformas con mayor riqueza de especies son la pendiente boscosa de la morrena adyacente al lago glaciar (Tabla 1, Bm1) con 27 especies, la morrena de fondo (Tabla $1, \mathrm{Bf}$ ) en proceso de colonización vegetal con 32 especies, y la turba de ciperáceas del cerro rocoso externo al valle glaciar (Tabla 1, Bc2) con 38 especies.

\section{GEOFORMAS PROGLACIARES DEL GLACIAR TYNDALL Y SU VEGETACIÓN}

Terrazas lacustres de la ribera del lago (Tabla 2, YL1, YL2)

Al borde del lago se encuentra una franja plana estrecha de hasta $10 \mathrm{~m}$ de ancho dominada por murtilla, Empetrum rubrum y ñire, Nothofagus antarctica (Tabla 2, YL1). El suelo de arenas gruesas está desvegetado en un 15\%. El estrato arbóreo tiene de 1 a $5 \mathrm{~m}$ de altura, el estrato arbustivo hasta 35 $\mathrm{cm}$, y el herbáceo hasta $15 \mathrm{~cm}$ de altura.

En la misma terraza alejándose del lago (Tabla 2,YL2), se encuentra el bosque dominado por lenga, Nothofagus pumilio. Las lengas tienen hasta $16 \mathrm{~m}$ de altura y de 15 a $45 \mathrm{~cm}$ de DAP. El estrato arbustivo es diverso pero de muy baja cobertura, la especie más frecuente es el chilco, Fuchsia magellanica. El sotobosque herbáceo tiene 100\% de cobertura y presenta señales de pastoreo vacuno reciente, la especie introducida Poa trivialis es la más abundante.

Similitud $0 / 0$

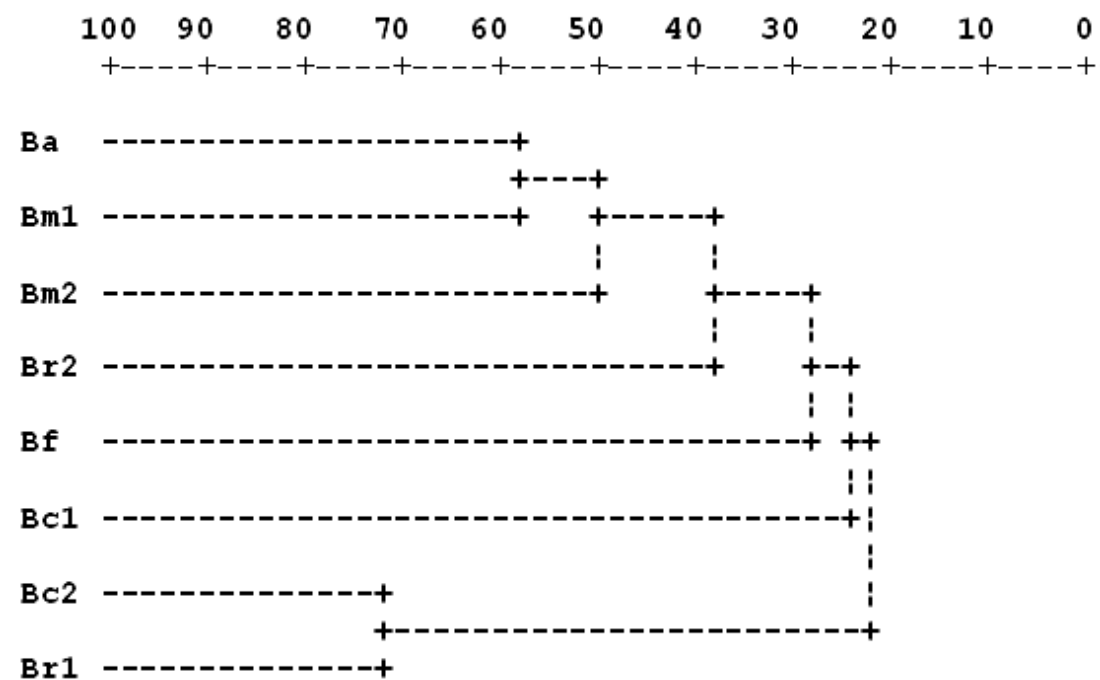

Fig. 2. Análisis de agrupamiento. Valores de similitud florística (\%), índice de Dice, entre las geoformas proglaciares del área del glaciar Balmaceda. 
Morrenas laterales (Tabla 2, Ym1, Ym2)

En las morrenas laterales y frontales más alejadas del glaciar (Tabla 2, Ym1) predomina el bosque de lenga, Nothofagus pumilio, con baja proporción de coihue, Nothofagus betuloides, son latizales de árboles establecidos posteriormente a un incendio. Los árboles alcanzan hasta $12 \mathrm{~m}$ de altura y hasta $20 \mathrm{~cm}$ de DAP. No hay estrato arbustivo, la cobertura de hierbas es entre 30 y $60 \%$.

En las morrenas laterales más cercanas al glaciar dominan los bosques de coihue (Tabla 2, Ym2), son también latizales post-incendio, los árboles alcanzan los $14 \mathrm{~m}$ de altura, y hasta $25 \mathrm{~cm}$ de DAP. No hay estrato arbustivo y las hierbas cubren hasta un $25 \%$.

Otras morrenas más recientes y depositadas a mayores elevaciones que las anteriores y cercanas al glaciar, presentan los inicios del proceso de colonización vegetal, distribuyéndose la vegetación en pequeños manchones herbáceos dispersos y aparecen los primeros árboles representados por el coihue, Nothofagus betuloides, el ñire, N. antarctica y el ciruelillo, Embothrium coccineum (Tabla 2, Ym2).

Depresiones intermorrénicas (Tabla 2, Yv1, Yv2)

En las depresiones intermorrénicas de amplitud variable con drenaje medio, se encuentra un bosque bajo y abierto de coihue, Nothofagus betuloides, con lenga, Nothofagus pumilio, ñire, N. antarctica, ciruelillo, Embothrium coccineum, y numerosas especies de arbustos como murtilla, Empetrum rubrum, chaura, Gaultheria mucronata, zarzaparrilla, Ribes magellanicum, chilco, Fuchsia magellanica, con un sotobosque herbáceo con especies higrófilas como Gunnera magellanica y especies de turberas como Schoenus antarcticus y Carpha schoenoides, también es abundante el musgo Racomitrium geronticum (Tabla 2, Yv1).

En las depresiones intermorrénicas mas amplias y mal drenadas entre los arcos morrénicos ubicados en el extremo del lago opuesto al glaciar (Tabla 2, Yv2), se encuentran turberas esfagnosas dominadas por el musgo Sphagnum magellanicum, con una flora herbácea típica Carex magellanica, Myrteola nummularia, Marsippospermum grandiflorum, Tetroncium magellanicum, Drosera uniflora, el subarbusto Lepidothamnus fonckii (Podocarpácea), y algunos pequeños árboles invasores de coihue, Nothofagus betuloides y ñire, N.antarctica.

\section{Morrena de fondo (Tabla 2, Yf)}

La morrena de fondo consiste en rocas sedimentarias aborregadas, recientemente abandonadas por el hielo, con muy baja cobertura vegetal, con depósitos glaciares muy delgados y dispersos que junto a las depresiones rocosas acumulan sedimentos, agua y semillas favoreciendo la colonización vegetal. En las depresiones rocosas con lagunas comienza a desarrollarse una comunidad turbosa con especies herbáceas como Carex banksii, Marsippospermum grandiflorum, Perezia lactucoides, y Rostkovia magellanica con arbolitos de hasta $60 \mathrm{~cm}$ de altura de las especies coihue, Nothofagus betuloides y ñire, Nothofagus antarctica y ocasionalmente ciruelillo, Embothrium coccineum (Tabla 2, Yf). En este ambiente se encuentran como especies pioneras los arbustos murtilla, Empetrum rubrum, romerillo, Chiliotrichum diffusum, calafate, Berberis microphylla, chaura, Gaultheria mucronata y diversas especies herbáceas muy dispersas en los roqueríos como Gunnera magellanica, Calceolaria tenella, Cerastium arvense y pteridófitas como Huperzia fuegiana y Lycopodium magellanicum.

\section{Cerros rocosos (Tabla 2, Yc)}

El cerro rocoso en la ribera sur del lago adyacente al hielo, tiene una altitud aproximada de $180 \mathrm{msnm}$ con un 30\% de roca desvegetada. En el cerro se encuentra un relicto del bosque de coihue, Nothofagus betuloides, los árboles antiguos alcanzan hasta los $12 \mathrm{~m}$ de altura y hasta $25 \mathrm{~cm}$ de DAP. Esta especie es muy combustible, el bosque fué incendiado y presenta una fuerte erosión del suelo. Al presente predominan los renovales de coihue, Nothofagus betuloides, con leñadura, Maytenus magellanica, canelo, Drimys winteri, ciruelillo, Embothirum coccineum y un brezal denso de chaura, Gaultheria mucronata con calafate, Berberis microphylla. La cubierta herbácea en ambientes húmedos está compuesta de Blechnum penna-marina, Gunnera magellanica, Ourisia ruelloides y en ambientes más secos Calceolaria biflora, Thlaspi magellanica, Draba magellanica, Senecio miser y otras especies (Tabla 2, Yf). 


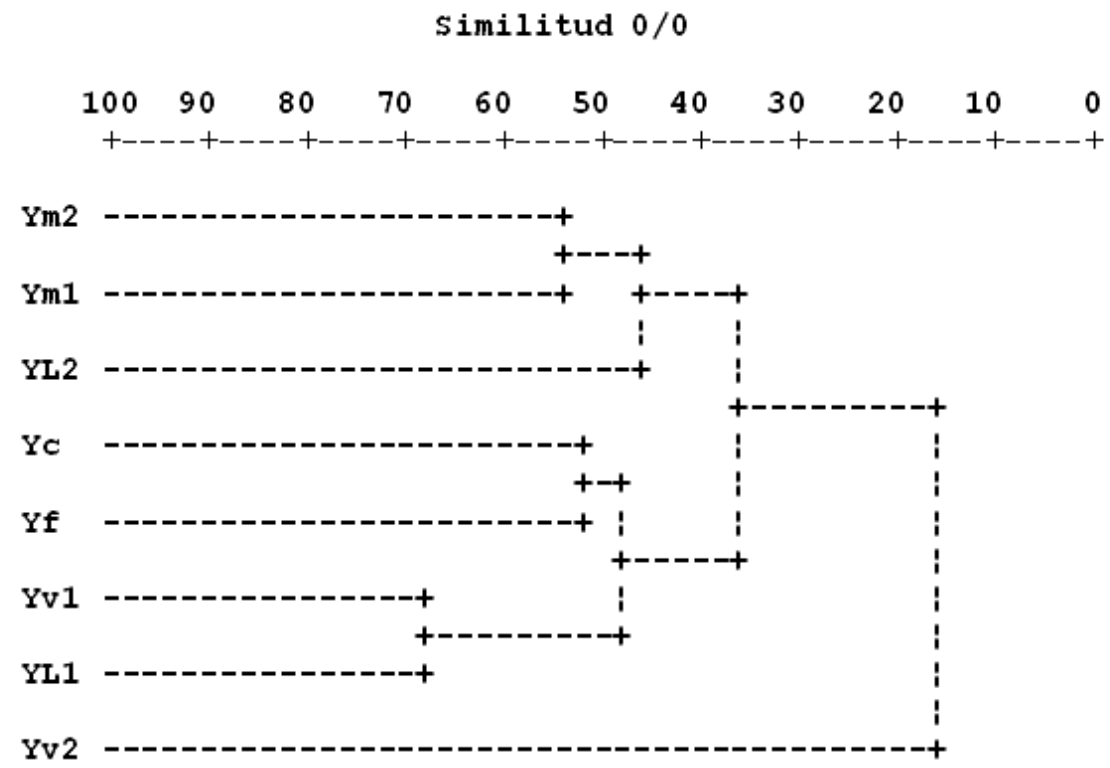

Fig. 3. Análisis de agrupamiento. Valores de similitud florística (\%), índice de Dice, entre las geoformas proglaciares del área del glaciar Tyndall.

Análisis de agrupamiento de la vegetación

El dendrograma (Fig. 3) de similitud florística a nivel del 40\% de similitud muestra tres grupos de geoformas : a) las morrenas (Tabla 2, Ym1, Ym2) y la terraza lacustre adyacente (Tabla 2, YL2) con bosques de lenga, Nothofagus pumilio, b) un grupo formado por dos subgrupos, el primero, de los ambientes rocosos del cerro con bosques de coihue, Nothofagus betuloides y chaura, Gaultheria mucronata y la morrena de fondo con vegetación herbácea dispersa e inicios de la colonización arbórea (Tabla 2, Yc y Yf), y el segundo, de las depresiones intermorrénicas con especies del bosque y de turbera, y la terraza lacustre en el borde del lago con bosquecillos de ñire (Tabla 2, Yv1 y YL1), c) un tercer grupo queda aislado y corresponde a una turbera esfagnosa de Sphagnum magellanicum, localizada en depresiones amplias entre los arcos morrénicos (Tabla 2, Yv2) con pequeños árboles invasores de coihue, Nothofagus betuloides y ñire, $N$. antarctica.

La riqueza de especies

En el glaciar Tyndall las geoformas con mayor riqueza de especies son la morrena de fondo (Tabla 2, Yf) con gran espacio disponible para la colonización con 37 especies. La depresión intermorrénica de drenaje medio (Tabla 2, Yv1) con 35 especies donde se produce una composición florística combinada de bosque y turba. La terraza lacustre (Tabla 2, YL2) adyacente a las morrenas laterales con 33 especies en el bosque de lenga.

\section{GEOFORMAS PROGLACIARES DEL GLACIAR TARABA Y SU VEGETACIÓN}

Arcos morrénicos (Tabla 3, Tm)

Los arcos morrénicos (Tabla 3, Tm) adyacentes al lago Taraba están cubiertos de un bosque de coihue, Nothofagus betuloides, abierto, con canelo, Drimys winteri y leñadura, Maytenus magellanica. El estrato arbustivo está dominado por chaura, Gaultheria mucronata y murtilla, Empetrum rubrum, y el piso del bosque está densamente cubierto por briófitas y helechos del género Hymenophyllum y son abundantes las especies herbáceas Gunnera magellanica y Lebetanthus myrsinites.

Los coihues, Nothofagus betuloides, alcanzan los $12 \mathrm{~m}$ de altura y $40 \mathrm{~cm}$ de DAP en los arcos morrénicos más antiguos. En la morrena mas reciente inmediata al lago glaciar, los coihues solo tienen hasta $6 \mathrm{~m}$ de altura. 
Depresiones intermorrénicas (Tabla 3, Tv).

Las depresiones intermorrénicas (Tabla 3, Tv) presentan un bosque de coihue, Nothofagus betuloides, muy abierto, donde dominan los arbustos murtilla, Empetrum rubrum y la chaura, Gaultheria mucronata. Los coihues alcanzan los $8 \mathrm{~m}$ de altura. El piso del bosque está totalmente cubierto por briófitas y son abundantes los helechos del género Hymenophyllum.

Aluviones intermorrénicos (Tabla 3, Ta)

En estos planos aluviales (Tabla 3, Ta) pedregosos, con arenas y gravas gruesas dominan bosquecillos de coihue, Nothofagus betuloides con arbolitos de hasta $5 \mathrm{~m}$ de altura, con un estrato arbustivo de murtilla, Empetrum rubrum y chaura, Gaultheria mucronata. Las briófitas son abundantes y también los helechos del género Hymenophyllum. Tienen una fisonomía muy similar a las depresiones intermorrénicas, pero se diferencian en el sustrato y en la composición florística, estos aluviones son permeables con mayor diversidad y abundancia de helechos y una notable mayor riqueza de especies.

Planos rocosos (Tabla 3, Tr)

En las planicies rocosas externas a los arcos morrénicos (Tabla 3, Tr), pulidas por los hielos, con suelos turbosos delgados, dominan las turberas de ciperáceas con las especies Schoenus antarcticus, Schoenus andinus, Carpha schoenoides, con especies pulvinadas como Astelia pumila, Donatia fascicularis, y algunos arbolitos de ciprés de las Guaitecas, Pilgerodendron uviferum, coihue, Nothofagus betuloides y ñire, $N$. antarctica.

\section{Cerros rocosos (Tabla 3, Tc)}

En las quebradas drenadas de los cerros, externas a los arcos morrénicos (Tabla 3, Tc), se encuentra un bosque muy abierto de coihue, Nothofagus betuloides, con ciprés de las Guaitecas, con canelo, Drimys winteri y con tepú, Tepualia stipularis y la trepadora coicopihue, Philesia magellanica. Los árboles alcanzan hasta $9 \mathrm{~m}$ de altura y hasta 25 $\mathrm{cm}$ de DAP. El sotobosque está totalmente cubierto de briófitas y son notables los helechos Blechnum magellanicum y Sticherus quadripartitus.

Análisis de agrupamiento de la vegetación

En el dendrograma (Fig. 4) por sobre el nivel del 60\% de similitud florística se forman tres grupos de geoformas: el primer grupo, con las morrenas (Tabla 3, Tm), las depresiones intermorrénicas (Tabla 3 , Tv) y los aluviones (Tabla 3, Ta) todos con bosques siempreverdes de coihue, Nothofagus betuloides. Un segundo grupo aislado que corresponde a los cerros rocosos (Tabla 3, Tc) con bosquecillos de coihue, Nothofagus betuloides con tepú, Tepualia

similitud $0 / 0$

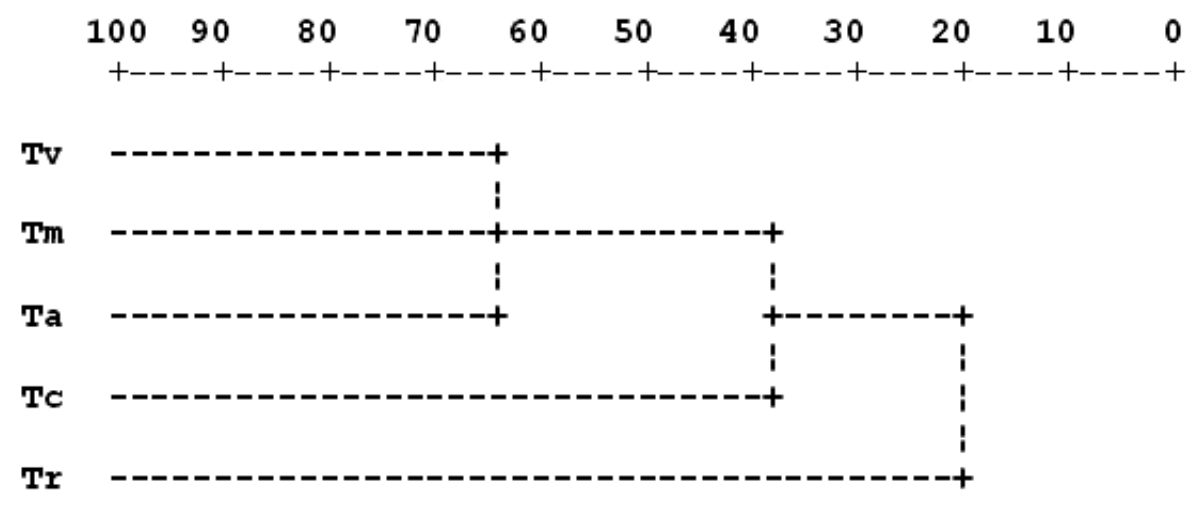

Fig. 4. Análisis de agrupamiento. Valores de similitud florística (\%), índice de Dice, entre las geoformas proglaciares del área del glaciar Taraba. 
stipularis y un tercer grupo también aislado de los planos rocosos (Tabla 3, Tr) con turberas de ciperáceas con especies de los géneros Schoenus y Carpha.

La riqueza de especies

En el valle glaciar Taraba la mayor riqueza de especies está en los bosques de coihue con murtilla de los aluviones (Tabla 3, Ta) con 35 sps., y en los bosques de coihue con chaura de las morrenas (Tabla $3, \mathrm{Tm})$ con $31 \mathrm{sps}$.

\section{GEOFORMAS PROGLACIARES DEL GLACIAR EMA Y SU VEGETACIÓN}

\section{Arcos morrénicos (Tabla 4, Em)}

El sistema de arcos morrénicos sucesivos ubicado en el frente del lago glaciar Ema, está cubierto con bosques de coihue, Nothofagus betuloides, con árboles de hasta $14 \mathrm{~m}$ de altura, desramados en un $90 \%$, y hasta $30 \mathrm{~cm}$ de DAP. El ñire, N. antarctica, es una especie frecuente y localmente se encuentra canelo, Drimys winteri. La chaura, Gaultheria mucronata es el arbusto más frecuente. El sotobosque está cubierto en un $50 \%$ aproximadamente con briófitas y son abundantes los helechos Himenofiláceos.

En la pendiente de la morrena más reciente, en el borde del lago glaciar, predomina un bosquecillo de ñire y es abundante la chaura, Gaultheria mucronata. Los ñires y coihues tienen de 2 a $3 \mathrm{~m}$ de altura.

\section{Depresiones intermorrénicas (Tabla 4, Ev)}

En el fondo de estrechas depresiones intermorrénicas (Tabla 4, Ev) con drenaje deficiente se forma un suelo turboso con bosque de coihue, Nothofagus betuloides y ñire, $N$. antarctica de cobertura variable y en el piso se encuentran herbáceas como Marsippospermum grandiflorum, Anthoxanthum redolens, Senecio acanthifolius, Gunnera magellanica.

\section{Aluviones del río Ema (Tabla 4, Ea1, Ea2)}

En los aluviones adyacentes y en el frente de los arcos morrénicos se encuentra un bosque de ñires, Nothofagus antarctica y coihues, Nothofagus betuloides en igual proporción (Tabla 4, Ea1), con coihues de hasta $12 \mathrm{~m}$ de altura y hasta $30 \mathrm{~cm}$ de DAP, y canelo, Drimys winteri, de hasta $6 \mathrm{~m}$ de altura. El estrato arbustivo es de baja cobertura con chaura, Gaultheria mucronata y michay, Berberis ilicifolia. El sotobosque está cubierto en un 100 \% con briófitas y son abundantes los helechos Himenofiláceos.

En el mismo aluvión, en el sector alejado de las morrenas y cercano al río Ema que desagua el lago glaciar, el bosque se tranforma gradualmente en un parque de ñire, Nothofagus antarctica, con un sotobosque turboso cubierto en un $100 \%$ con briófitas donde son especies frecuentes el junquillo, Marsippospermum grandiflorum, Myrteola nummularia, Gunnera lobata, y ciperáceas como Schoenus antarcticus, Carpha schoenoides.

\section{Cerros rocosos (Tabla 4, Ec1, Ec2)}

En las hondonadas y peldaños rocosos de los cerros externos a los arcos morrénicos (Tabla 4, Ec1), se encuentran bosques siempreverdes de coihue, Nothofagus betuloides y canelo, Drimys winteri, con árboles bajos deformados por el viento de hasta $6 \mathrm{~m}$ de altura, el sotobosque en gran parte cubierto de briófitas, con helechos Himenofiláceos y Blechnum magellanicum y Sticherus quadripartitus .

En las laderas inferiores de los cerros y plano rocoso (Tabla 4, Ec2) predominan las turberas de ciperáceas, dominadas por Schoenus antarcticus con especies pulvinadas, como Donatia fascicularis, Astelia pumila, Caltha dioneifolia, y numerosas especies propias de este ambiente Tribeles australis, Oreobolus obtusangulus, Drosera uniflora, Gaimardia australis, Tetroncium magellanicum, Tapeinia magellanica, Gunnera lobata, Phyllachne uliginosa y otras.

Análisis de agrupamiento de la vegetación

En el dendrograma (Fig. 5) por sobre el nivel del 40\% de similitud florística, se separan dos grupos de geoformas: el primer grupo, lo forman las turberas de ciperáceas dominados por Schoenus antarcticus que cubren el aluvión (Tabla 4, Ea2) $y$ los cerros rocosos (Tabla 4, Ec2). En el segundo grupo, se unen escalonadamente los bosques de coihue, Nothofagus betuloides que dominan los 


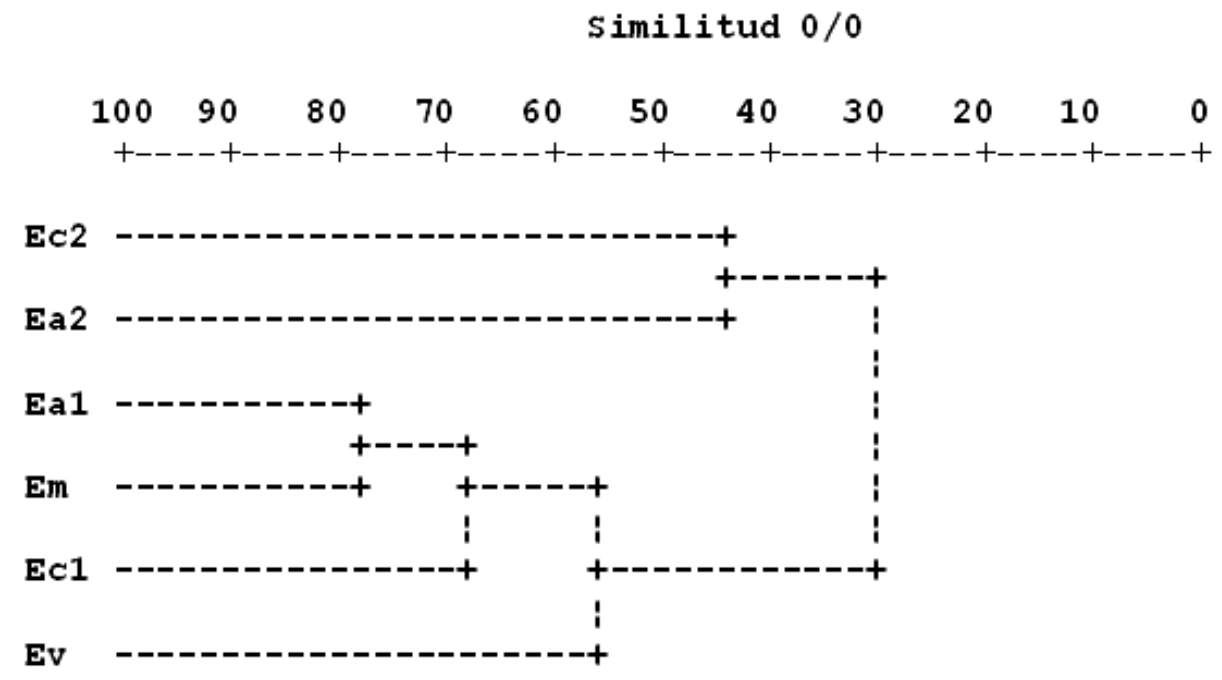

Fig. 5. Análisis de agrupamiento. Valores de similitud florística (\%), índice de Dice, entre las geoformas proglaciares del área del glaciar Ema.

arcos morrénicos (Em), el aluvión adyacente a los mismos (Ea1), las hondonadas rocosas de los cerros (Ec1) y las depresiones intermorrénicas (Ev). Los bosques de los arcos morrénicos (Em) y el aluvión adyacente a los mismos (Ea1) son muy similares florísticamente, casi un $80 \%$.

La riqueza de especies

El bosque de coihue (Tabla 4, Ec1) y la turbera de ciperáceas (Tabla 4, Ec2) que cubren los cerros rocosos tienen la mayor riqueza de especies, 25 especies. También el parque de ñire del aluvión (Tabla 4, Ea2) con suelo turboso tiene 24 especies.

\section{DISCUSIÓN}

Este estudio representa una descripción vegetacional intantánea de los cuatro valles glaciares estudiados, dentro del proceso de sucesión vegetal de cada uno de ellos. Cada valle glaciar tiene características físicas y geográficas e historia glaciar distintas por lo que se esperan también diferencias florísticas en la sucesión vegetacional entre ellos como muestran los resultados. Sin embargo, el climax vegetacional final tiende a ser muy similar para los cuatro valles glaciares por la presencia y abundancia actual de las especies dominantes constructoras de comunidaes climax ya conocidas regionalmente.

Las formaciones de bosques siempreverdes de coihue, Nothofagus betuloides y las turberas de ciperáceas, Schoenus antarcticus y de esfagno, Sphagnum magellanicum, que cubren los cerros y planos rocosos externos a los arcos morrénicos y que flanquean los valles glaciares, se corresponden con la comunidades vegetales climáxicas ya descritas para la región de Magallanes por Pisano (1977) por haber sido espacios abandonados por el hielo de muy antiguo.

Las formaciones vegetales de los arcos morrénicos, depresiones intermorrénicas, aluviones, terrazas lacustres y morrenas de fondo, todas geoformas mas recientes dentro del valle glaciar, están cubiertas con comunidades vegetales en distintos estadios sucesionales.

En todas estas formaciones la sucesión se dirige hacia el climax de los bosques de Nothofagus en los sustratos con buen drenaje, morrenas, aluviones, terrazas lacustres, o hacia el climax de las turberas de ciperáceas o de Sphagnum en los sustratos con drenaje deficiente como en las depresiones intermorrénicas con arcillas, o material morrénico con arcillas encerrado entre arcos morrénicos (Pisano, 1977).

El glaciar Tyndall en particular, dada su posición más continental (Henríquez, 2002), respecto a los otros tres glaciares, no tiene turberas de ciperáceas, 
Schoenus antarctica, y si tiene bosques de lenga, Nothofagus pumilio. Estos se ubican en los arcos morrénicos de la parte media y extrema del lago glaciar. La lenga no es pionera en los ambientes periglaciares y sus bosques representarían un estadio tardío de la sucesión (Armesto et al., 1992).

En los valles glaciares la riqueza de especies mayor tiende a estar en las morrenas de fondo. Sobre la superficie rocosa pulida hay numerosos micrositios como depresiones rocosas (Pisano 1971) con o sin agua, acumulación de piedras y gravas que capturan sedimentos, agua y semillas y donde se establecen las primeras especies. Las primeras especies arbóreas que se establecen son el coihue, Nothofagus betuloides, el ñire, Nothofagus antarctica y el notro o ciruelillo, Embothrium coccineum coincidiendo con Sweda (1987). En las depresiones rocosas con lagunas aparecen los primeros parches de turberas con los árboles mencionados y especies como el junquillo, Marsippospermum grandiflorum, Carex banksii, Rostkovia magellanica, Epilobium australe, Deschampsia antarctica, Perezia lactucoides propias de ambientes húmedos.

\section{LITERATURA CITADA}

ANIYA, M. 1985. Landforms in the proglacial area of Soler Glacier. En: "Glaciological Studies in Patagonia Northern Icefield". Edit. C. Nakajima (Data Center for Glacier Research, Japan) 80-87

ARMESTO, J., I. CASSASA \& O. DOLLENZ 1992. Age estructure and dynamics of Patagonian beech forests in Torres del Paine National Park, Chile. Vegetation, 98: 13-22

BURROWS, C. 1990. Processes of Vegetation Change. Unwin Hyman, Wellington, New Zealand., pp 551.

DOLLENZ, O. 1991. Sucesión vegetal en el sistema morrénico del Glaciar Dickson, Magallanes, Chile. Anales Instituto de la Patagonia, (Chile), 20: 49-60

DOMÍNGUEZ, E., E. PISANO \& O. DOLLENZ 1999. Colonización vegetal en el área periglaciar del glaciar Nueva Zelandia, Cordillera Darwin de Tierra del Fuego, Chile. Anales Instituto de la Patagonia, (Chile) 27: 7-16
FUJIYOSHI, Y., H. KONDO, J. INOUE \& T. YAMADA 1987. Characteristics of precipitation and vertical structure of air temperature in the Northern Patagonia. Bulletin of Glacier Research, 4: 15-23

HENRÍQUEZ, J.M. 2002. Análisis de la flora vascular de valles glaciares de la región de Magallanes, Chile. Anales Instituto de la Patagonia, 30:25-40

HENRÍQUEZ, J.M. \& N. CAAMAÑO 2002. Estudios liquenométricos en el glaciar Nueva Zelandia, Tierra del Fuego: datos preliminares. Anales Instituto de la Patagonia, 30: 41-48

MOORE, D.M. \& E. PISANO 1997. Biotic colonization of recently deglaciated areas in FuegoPatagonia: Phytogeographical considerations. Anales Instituto de la Patagonia, (Chile), 25: $21-46$

MUELLER-DOMBOIS, D. y H. ELLENBERG, 1974. Aims and methods of vegetation ecology. John Wiley \& Sons. New York. 574 pp.

NAVARRO, R. 1984. Programa computacional para el análisis numérico de comunidades: diversidad y sobreposición. Medio Ambiente, 7: $82-87$

PISANO, E. 1971. Comunidades vegetales del área del fiordo Parry, Tierra del Fuego, Parque Nacional "Alberto de Agoistini". Anales Instituto de la Patagonia, (Chile) 1-2: 93-133

PISANO, E. 1977. Fitogeografía de Fuego-Patagonia chilena. I. Comunidades vegetales entre las latitudes $52^{\circ}$ y $56^{\circ}$ Sur. Anales Instituto de la Patagonia, (Chile) 8: 121-250

PISANO, E. 1978. Establecimiento Nothofagus betuloides (Mirb.) Blume (Coigüe de Magallanes) en un valle en proceso de desglaciación. Anales Instituto de la Patagonia, (Chile) 9: $107-128$

STRELIN, J., CASASSA, G., G. ROSKVIST \& P.HOLMLUND 2008. Holocene glaciations in the Ema Glacier Valley, Monte Sarmiento Massif, Tierra del Fuego. Palaeogeography, Palaeoclimatology, Palaeoecology, 260: 299-314.

SWEDA, T. 1987. Recent retreat of Soler glaciar, Patagonia, as seen from vegetation recovery. Bulletin of Glacier Research, 4: 119-124 
